\title{
Performance analysis of dual-pump nonlinear amplifying loop mirror mode-locked all-fibre laser
}

\author{
Sonia Boscolo ${ }^{1}$, Christophe Finot ${ }^{2}$, Ilya Gukov ${ }^{3}$ and Sergei \\ K Turitsyn ${ }^{1,4}$ \\ 1 Aston Institute of Photonic Technologies, School of Engineering and Applied \\ Science, Aston University, Birmingham B4 7ET, UK \\ ${ }^{2}$ Laboratoire Interdisciplinaire Carnot de Bourgogne, UMR 6303 Université de \\ Bourgogne-Franche-Comté - CNRS, 9 avenue Alain Savary, Dijon, France \\ 3 Moscow Institute of Physics and Technology \& Skolkovo Institute of Science \\ and Technology, Moscow, Russia \\ 4 Aston-NSU International Centre for Photonics, Novosibirsk State University, \\ Novosibirsk, 630090, Russia \\ E-mail: s.a.boscolo@aston.ac.uk
}

\begin{abstract}
We numerically characterise, in the three-dimensional space of adjustable cavity parameters, the performance of a recently reported layout of a flexible figure- 8 laser having two independently pumped segments of active fibre in its bidirectional ring [1]. We show that this optimisation problem can be efficiently addressed by applying a regression model based on a neural-network algorithm.
\end{abstract}

Keywords: mode-locked fibre lasers, fibre optics amplifiers and oscillators, nonlinear optics 


\section{Introduction}

At the level of fundamental research, mode-locked lasers constitute an ideal platform for the investigation of original and complex nonlinear dynamics of ultrashort pulses, while with regard to applications, pulses with different and optimised features are sought with the general constraint of developing efficient cavity architectures. In both these aspects, fibre laser systems, which offer compact and versatile design, represent an area of intense research and development [2]. One of the earliest reported passively mode-locked laser schemes - the figure-8 (F8) laser [3] - is currently receiving renewed interest as a reliable, robust and lowcost all-fibre ultra-fast source [4]. F8 lasers consist of a bidirectional and unidirectional ring. The bidirectional ring forms a loop mirror that is power imbalanced (either actively or passively) to induce a differential phase, and consequently a power-dependent reflectivity that mimics the action of a saturable absorber [5], promoting pulse generation in the main laser cavity. Unlike fibre lasers using the nonlinear-polarisationevolution (NPE) effect, F8 lasers can be designed in an all-polarisation-maintaining configuration, thereby further improving their environmental stability and robustness [6]. The use a nonlinear amplifying loop mirror (NALM) as the mode-locking element has enabled both stable all-normal-dispersion lasers in the $1-\mu \mathrm{m}$ emission band of ytterbium $[7,8]$ and large net-normal dispersion lasers in the 1.55$\mu \mathrm{m}$ emission band of erbium [9] to produce highenergy pulses that are externally compressible to several hundreds femtosecond duration. While several parameters determine the performance of standard F8 lasers with a single unbalancing element in their bidirectional ring, once the laser is assembled, the reflectivity/transmittance of the fibre loop mirror depends only on the intra-cavity radiation, and the only degree of freedom of the system is the optical power level. Although the latter can be adjusted over a relatively wide range in some configurations, this results in energy scaling at virtually constant peak power [10]. Recently, a new design of a F8 laser employing a NALM with two active fibre segments and two independently controlled pump-power modules has been proposed and experimentally demonstrated $[1,11]$. This laser layout combines the reliability and robustness of conventional F8 lasers with the flexibility of NPE-based lasers, providing access to a variety of generation regimes with a relatively wide adjustment range of the main pulse parameters. Moreover, it enables reliable and reproducible live electronic adjustment of the lasing regimes [12], which is practically impossible to do by adjusting fibre-based polarisation controllers in NPE-based lasers. Indeed, as in this laser configuration the generation properties are practically independent of the ambient conditions, it is only necessary to determine once, initially, the required values of the ratio of the pump powers and the ranges of their adjustment. After that, the laser will reliably reproduce the expected generation parameters. In comparison with a double NALM laser configuration where the second independently pumped active medium is added to the unidirectional ring of the F8 laser [4, 9], the dual-pump NALM architecture can deliver higher average and peak radiation powers [13].

In this Letter, we numerically explore the broad space of operating states of the dual-pump NALM laser that can be accessed through independent control of the pump powers in the two gain segments and the laser output coupling ratio, which is an additional easy-to-control parameter that impacts the operating states of the laser. The general problem of optimisation towards a target operational regime in a complex multi-parameter space can be intelligently addressed by implementing machine learning strategies. Evolutionary algorithms and neural networks (NNs) have been recently applied in ultrafast fibre lasers to automate the turn-on procedure, and determine and control parameters to maintain desired modes of operation $[14,15,16,17]$. In this work, we use a regression model based on a NN algorithm for an automated exploration of the cavity parameter space and a rapid identification of modelocked solutions. It is worth noting that the electronic control over the parameters of the generated pulses that is afforded by the NALM laser architecture is exempt from a complicated computer search of suitable regimes during laser operation [12]. Correspondingly, here we use the NN only to quickly figure out the output pulse features that are attainable through variation of the adjustable system parameters.

\section{Laser model and numerical procedure}

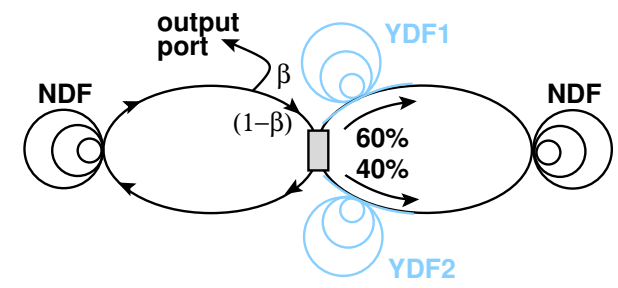

Figure 1. Schematic of the laser. YDF: ytterbium-doped fibre; NDF: normally dispersive fibre.

We consider a fibre oscillator similar to the experimental setup in [1] as shown in figure 1. The laser consists of a passive unidirectional ring and an active bidirectional loop (known as NALM) connected via a 60/40 coupler. The passive ring includes a 
$2.8 \mathrm{~m}$-long section of single-mode normally dispersive fibre (NDF) and a $\beta /(1-\beta)$ fibre output coupler, while the NALM contains two sections of ytterbiumdoped fibre, YDF1 and YDF2, of respective lengths $5.3 \mathrm{~m}$ and $5.0 \mathrm{~m}$, separated by a $12.4 \mathrm{~m}$-long section of NDF. The cavity group-velocity dispersion (GVD) is $0.58 \mathrm{ps}^{2}$, indicating that the dissipative soliton shaping is expected. We model the laser cavity by evolving the intra-cavity pulse dynamics in a component by component manner. In the fibres, propagation follows a standard modified nonlinear Schrödinger equation (NLSE) for the slowly-varying pulse envelope:

$i \psi_{z}-\frac{1}{2} \beta_{2} \psi_{t t}+\gamma|\psi|^{2} \psi=\frac{i}{2} g(z, \omega) \psi$,

where $\beta_{2}$ is the GVD parameter, $\gamma$ is the Kerrnonlinearity coefficient, and $g$ is the gain coefficient. The latter is null for the passive fibres, considered lossless. In the YDFs, the gain $g$ includes saturation and spectral response, as $g(z, \omega)=g_{m}(z) g_{p}(\omega)$. The amplitude of the gain coefficient $g_{m}(z)$ is dependent on the average signal and pump powers through $g_{m}(z)=(1 / P(z))(\mathrm{d} P / \mathrm{d} z)(P(z)$ is the signal power $)$, the average power dynamics being described by standard steady-state rate equations for an effective two-level system $[18,19]$. These are solved at each fibre longitudinal step, and subsequently the gain coefficient amplitude is evaluated at that step. Finally, implementing the spectral response to recover $g(z, \omega)$ allows for the use of the gain in the splitstep integration of (1). We note that this stage is computationally demanding under standard computer resources mainly due to the bi-directional pumping configuration. The signal and pump saturation powers and absorption coefficients are calculated using the material and fibre parameters given in [19]. A similar numerical model of the laser based on a NLSE was used and qualitatively validated in [1].

In a first approach we perform numerical simulations of the propagation model (1) using parallel computing on the CPU of a standard personal computer. We use a grid of $7 \times 8 \times 7$ points to discretise the parameter space of output coupling factor $\beta$, total pump level delivered to the active fibres $P_{\mathrm{T}}=P_{p, 1}+P_{p, 2}$, and fractional pump power $R=P_{p, 1} / P_{\mathrm{T}}$, and for each point we record the output pulse characteristics for single-pulse stationary mode-locked states. The variation ranges of $\beta, P_{\mathrm{T}}$ and $R$ used are: 0.1 to $0.9,2.5 \mathrm{~W}$ to $11.0 \mathrm{~W}$, and 0.1 to 1.0, respectively. However, such a study based on extensive numerical simulations is a costly exercise, the computation of each point requiring approximately 2 hours. Therefore, in a second approach we use a NN based model trained on the data from numerical simulations to make predictions across a larger range of the data domain. The algorithm handles almost instantly the whole parameter space. We use a feed-forward NN relying on the Levenberg-Marquardt back propagation algorithm and involving three hidden layers. The number of hidden neurons (around 10 in each layer) is determined by a rule-of-thumb method to prevent overfitting. This NN is a part of the Matlab NN toolbox. It is worth noting here that the experimental data that are available in the literature [1] represent a limited set of data in the three-dimensional space of $\left(\beta, P_{\mathrm{T}}, R\right)$, especially pertaining to the output coupling factor $\beta$. On the other hand, ad hoc numerical tools can advantageously be used to cover the entire parameter space.

\section{Results and discussion}

\subsection{Direct numerical simulations}

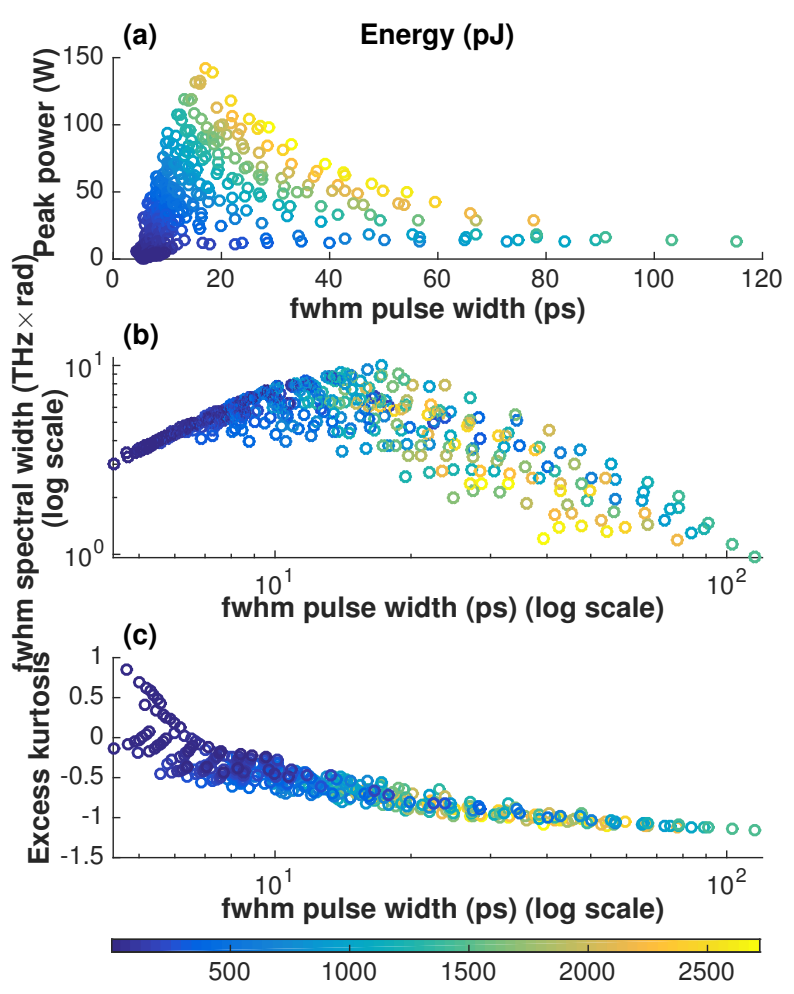

Figure 2. Scatter plots of output pulse characteristics from the laser as obtained from NLSE numerical simulations for variable $\left(\beta, P_{\mathrm{T}}, R\right)$ parameters. (a) Pulse peak power, (b) full-width at half-maximum (fwhm) spectral width, and (c) excess kurtosis as a function of fwhm pulse width. The points are colour-coded to display the pulse energy.

Figure 2 shows scatter charts of various characteristics of the output pulses from the laser as obtained from numerical simulations of the governing equations for the set of input parameters $\left(\beta, P_{\mathrm{T}}, R\right)$ described in the previous section. We note that around $5 \%$ of these simulations did not lead to single-pulse period- 

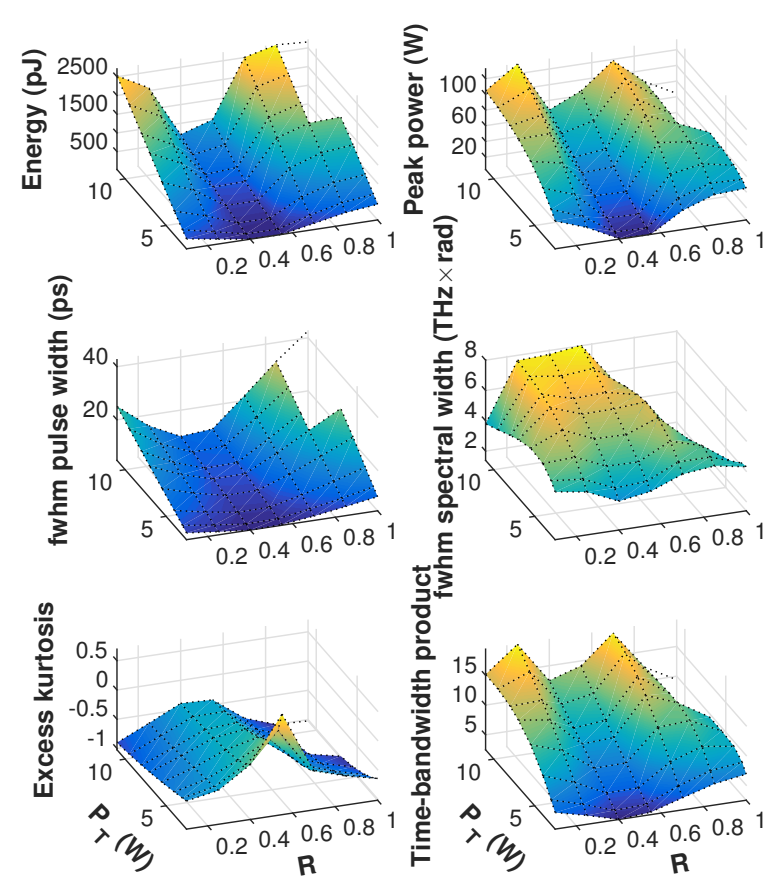

Figure 3. Maps of the output pulse energy, peak power, fwhm temporal and spectral widths, excess kurtosis and timebandwidth product in the two-dimensional space of total and fractional pump powers for $\beta=0.75$, as obtained from NLSE numerical simulations.
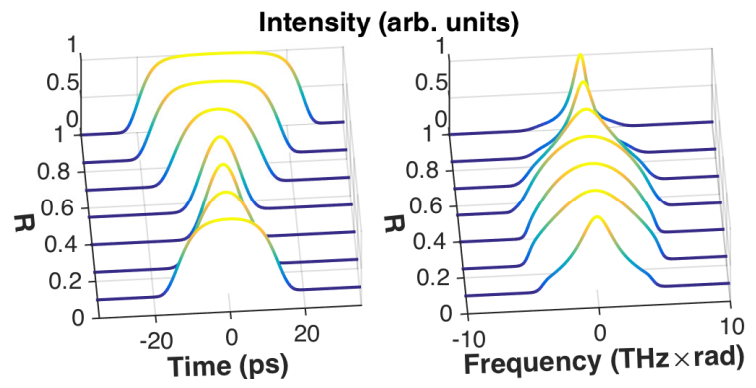

Figure 4. Evolution of the pulse temporal and spectral intensity profiles with $R$ for $\beta=0.75$ and $P_{\mathrm{T}}=11.0 \mathrm{~W}$.

one steady states of the laser, but multi-pulse mode locking or, in a few instances, also period doubling was observed instead. The corresponding output data values are excluded from figure 2. Some interesting correlations among the output pulse parameters are revealed by the plots in figure 2. Firstly, an increase in pulse energy stemming from a higher level of pump power is generally achieved through a combination of peak power increase and pulse elongation in different proportions (figure 2a). This corroborates the possibility of scaling the pulse peak power with the energy offered by the dual-pump NALM configuration, in agreement with the results presented in [1]. Remarkably, while the total pump power delivered to the active fibres is varied here by less than $7 \mathrm{~dB}$, the pulse peak power and temporal width display potential variation ranges of nearly $25 \mathrm{~dB}$ and $15 \mathrm{~dB}$, respectively. The principle of peak power adjustment within the dual-pump NALM laser scheme can be readily understood from consideration of the continuous-wave power transfer function of the nonlinear fibre loop [5]. Indeed, the condition of mode-locked laser operation at the maximum of the NALM transmission curve [3] yields a pulse peak power of $P=\pi /\left[\gamma L\left(\alpha g_{1}-(1-\alpha) g_{2}\right)\right]$, where $\alpha$ is the coupling coefficient of the NALM, $L$ and $\gamma$ are the respective length and nonlinearity coefficient of the passive fibre inside the NALM, $g_{1}$ and $g_{2}$ are the amplification coefficients of the two active fibres, and their product $g_{1} g_{2}$ compensates for optical losses at the steadystate operation of the laser. Because $g_{1}$ and $g_{2}$ can be varied independently keeping their product $g_{1} g_{2}$ constant, the pulse peak power becomes tuneable [1]. Figure 2(b) reveals that for pulses of low energy there is a definite power relationship between temporal duration and spectral bandwidth. This clearly indicates that the time-bandwidth product of the pulses is not preserved, that is, the pulses are far from their Fourier-transform limit. As a measure of the pulse shape we use here the excess kurtosis, defined as $\mu_{4} / \sigma^{4}-3$, where $\mu_{4}$ and $\sigma$ are the fourth order central moment and standard deviation of the pulse intensity profile, respectively, and 3 is the kurtosis of a Gaussian profile [20]. Shapes with a positive excess kurtosis have long and fat tails relative to a Gaussian shape, whereas negative excess kurtosis equals shorter and thinner tails than the Gaussian profile. As we can see in figure 2(c), low-energy pulses feature positive or slightly negative excess kurtosis, while the excess kurtosis becomes increasingly more negative with increasing pulse energy. The very large range of excess kurtosis values that are observed, from -1.15 to 0.85 , indicates that a broad variety of pulse shapes can be obtained directly at the laser output without the need for external shaping. However, a comparison between figure $2 \mathrm{~b}$ and figure $2 \mathrm{c}$ reveals that it is not possible to achieve a given pulse shape with any pulse duration and spectral width, but there are combinations of temporal and spectral widths that cannot be realised even within the large flexibility of this laser architecture.

A closed-up view of the pulse generation regimes of the laser is provided by figure 3 , which shows maps of the typical output pulse characteristics in the twodimensional parameter space of total and fractional pump powers in the active fibres for a particular value of the output coupling coefficient $(\beta=0.75)$. The maps shown are representative of those observed for the other $\beta$ values too, for which the pulse parameters evolve in a closely similar fashion with the pump power 

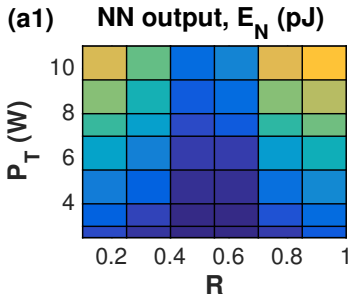

(a3)

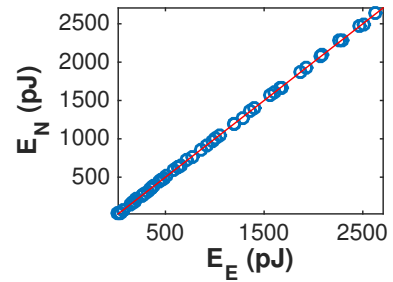

(a2) Expected values, $E_{E}$ (pJ)

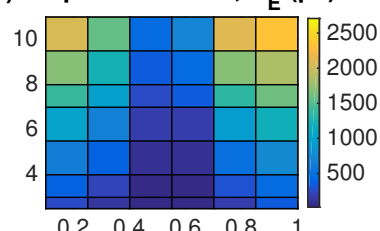

(a4) Error, $\left(E_{E}-E_{N}\right) / E_{E}(\%)$

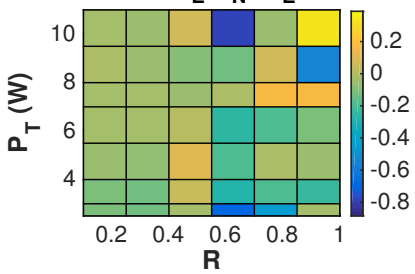

(b1) NN output, $E_{N}$ (pJ)

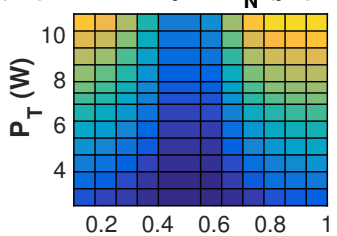

(b3) Expected values, $\mathrm{E}_{\mathrm{E}}$ (pJ) (b4)
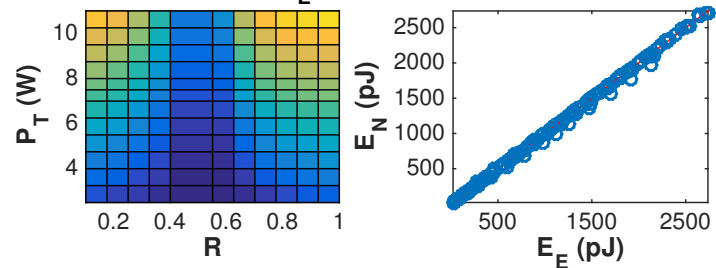

Figure 5. Extrapolation performance of the NN. (a) Predictions on a new value of the output coupling coefficient $(\beta=0.7)$. The two-dimensional map of energy values generated by the NN (panel 1) is compared to the map of expected values produced by NLSE numerical simulations (panel 2). Panel 3 and 4 show the regression between NN output and expected values, and the map of relative error values, respectively. (b) Predictions on new values of the total and fractional pump powers for $\beta=0.75$. The two-dimensional map of energy values generated by the NN (panel 1) is compared to the map of expected values produced by NLSE numerical simulations (panel 3). Also shown are the training data (panel 2) and the regression between NN output and expected values (panel $4)$.

parameters, though quantitative differences exist. We can see that the pulse energy increases with $P_{\mathrm{T}}$ at a fixed $R$ as expected. The peak power of the pulses features a similar functional dependence on $P_{\mathrm{T}}$ for a given $R<1$, whereas it remains approximately constant at varying levels of total pump power when $R=1$, which corresponds to the case of a conventional NALM configuration with a single active fibre $[1,10]$. It is noteworthy to highlight that the evolution of the pulse parameters with the fractional pump power at a fixed $P_{\mathrm{T}}$ is approximately symmetric about $R$ slightly less than 0.5 , and the pulse energy and peak power reach their minimum values at such a point, in accordance with the NALM properties. Indeed, for such a value of $R$ the gains $g_{1}$ and $g_{2}$ of the two active fibres are approximately equal, and so the differential power level, hence differential nonlinear phase shift between the two counter-propagating signals in the NALM loop is provided only by the asymmetric loop coupler. The map of time-bandwidth product values confirms that the laser pulses are chirped. Importantly, varying the ratio of pump power levels in the gain segments provides access to a variety of pulse generation regimes that differ from each other not only quantitatively in terms of main pulse parameters, but also qualitatively as well. Indeed, as figure 4 shows, slight tuning of the pump power ratio at a fixed total pump power enables the generation of pulses with parabolic or flat-top temporal waveforms from the laser cavity. Accordingly, this new dual-pump NALM laser architecture provides a somewhat alternative approach for nonlinear pulse shaping to other fibre laser schemes that have been recently suggested [21, 22]. All pulses generated in this laser come with a fairly linear chirp. We have explored the compressibility of the pulses by de-chirping them with a linear dispersive delay line. For the pulses shown in figure 4, compression factors from more than 15 to almost 35 are achievable.

\subsection{Neural-network regression}

We have evaluated the ability of the regression model to make good predictions, or at least not to fail catastrophically, when the training data covers only a limited range of the data domain. The training of the NN was restricted to the data obtained for the same parameter ranges as those used in the numerical simulations of the NLSE propagation model (described in section 2). The regression plots resulting from interpolation from the training data set to previously unseen data were well fitted with a correlation of almost $100 \%$ for all the output pulse parameters. We have then asked the network to make predictions for a new value of the output coupling coefficient $(\beta=$ 0.7). The extrapolation generalisation performed by the network on the output pulse energy is illustrated in figure $5 \mathrm{a}$. The predictions from the regression model show good quantitative agreement with the results of simulations of the governing equations carried out over the new parameter domain, with a percent error that does not exceed 1\%. Percent errors of less than $1 \%$ were also achieved for the other pulse parameters. Another illustration of the extrapolation performance of the NN is provided by figure $5 \mathrm{~b}$. This time we have asked the network to extrapolate from the training 
data set to new data corresponding to a more resolved grid of points in the parameter space of total and fractional pump power for a given $\beta$ value. Again, there is is good quantitative agreement between the predictions from the regression model and the results of NLSE simulations carried out over the extended parameter domain. The percent error did not exceed $15 \%$ for the pulse energy and peak power, and was less than $5 \%$ for the pulse temporal and spectral widths. These results confirm that the NN model can greatly accelerate the solution of the multi-parameter optimisation problem being considered, compared to direct numerical simulations. Further, the model could be used in a combination with plots of characteristic surfaces in the space of system parameters obtained from low-resolution direct numerical simulations to quickly identify the optimal working parameters for the generation of pulses with prescribed features [23].

\section{Conclusion}

Mode-locked fibre lasers possess the unique potential of a large variety of lasing regimes, not only using different cavity configurations, but also in a single cavity layout through variation and adjustment of its parameters. In this paper, we have presented an indepth numerical characterisation of the various pulse generation regimes of a novel NALM laser with a second segment of active fibre and corresponding pump source, which can be accessed through independent control of the pump powers in the two gain segments and the laser output coupling ratio. We have shown that the application of an NN-based regression model provides a rapid and precise identification of the attainable output pulse properties. Combined with a graphical representation of characteristic surfaces in the space of system parameters, our NN method could provide a sufficient resolution to guide the choice of the parameters that are required to achieve a given pulse target [23]. At this stage the algorithm cannot detect multi-pulse or multi-period modelocking regimes, which are occasionally observed in the laser. Notwithstanding, one can advantageously use the extrapolation generalisation performed by the $\mathrm{NN}$ to determine the output parameter space that is accessible, and then perform refined numerical simulations of the governing equations over the input parameter subsets of interest to unveil the physical behaviour of the system. Our approach opens new opportunities for exploring nonlinear cavity dynamics in laser systems where the vast parameter space makes systematic exploration impracticable, yet ideally suited to optimisation by a NN algorithm. Therefore, it confirms the recent interest in the use of $\mathrm{NN}$ algorithms for handling complex optical structure designs [24], ultrafast optics processes [25] or complex nonlinear problems [26]. Future work may consider the upgrade of the NN model for handling multi-pulse and multiperiod mode-locking operation. Finally, combining the NN analysis with an appropriate feedback loop will pave the way for a smart self-tuning laser system.

We acknowledge the support of the Institut Universitaire de France and the Russian Science Foundation (Grant No. 17-72-30006).

\section{References}

[1] Smirnov S, Kobtsev S, Ivanenko A, Kokhanovskiy A, Kemmer A and Gervaziev M 2017 Opt. Lett. 42 1732-35

[2] Nyushkov B N and Kobtsev S M 2018 Quantum Electron. 48 1099-1104

[3] Duling I 1991 Opt. Lett. 16 539-41

[4] Runge A F J, Aguergaray C, Provo R, Erkintalo M and Broderick N G R 2014 Opt. Fiber Technol. 20 657-65

[5] Doran N and Wood D 1988 Opt. Lett. 13 56-8

[6] Fermann M E and Hartl I 2013 Nat. Photon. 7 868-74

[7] Aguergaray C, Hawker R, Runge A F J, Erkintalo M and Broderick N G R 2013 Appl. Phys. Lett. 103121111

[8] Erkintalo M, Aguergaray C, Runge A F J and Broderick N G R 2012 Opt. Express 20 669-674

[9] Bowen P, Erkintalo M and Broderick N G R 2018 Opt. Commun. 410 447-451

[10] Lin H, Guo C, Ruan S and Yang J 2014 Laser Phys. Lett. 11085102

[11] Ivanenko A, Kobtsev S, Smirnov S and Kemmer A 2016 Opt. Express 24 6650-55

[12] Kobtsev S, Ivanenko A, Kokhanovskiy A and Smirnov S 2018 Laser Phys. Lett. 15045102

[13] Ivanenko A V, Kokhanovsky A Y, Gervaziev M D, Smirnov S V, Kobtsev S M and Turitsyn S K 2018 Proc. of SPIE vol 10811, High-Power Lasers and Applications IX $\mathrm{p} 1081117$

[14] Fu X and Kutz J N 2013 Opt. Express 21 6526-37

[15] Woodward R I and Kelleher E J R 2016 Sci. Rep. 637616

[16] Baumeister T, Brunton S L and Kutz J N 2018 J. Opt. Soc. Amer. B 35 617-26

[17] Andral U, Buguet J, Si Fodil R, Amrani F, Billard F, Hertz E and Grelu P 2016 J. Opt. Soc. Amer. B 33 825-833

[18] Pask H M, Carman R J, Hanna D C, Tropper A C, Mackechnie C J, Barber P R and Dawes J M 1995 IEEE J. Sel. Top. Quantum Electron. 1 2-13

[19] Turitsyn S K, Bednyakova A E, Fedoruk M P, Latkin A I, Fotiadi A A, Kurkov A S and Sholokhov E 2011 Opt. Express 19 8394-8405

[20] DeCarlo L T 1997 Phychol. Methods 2 292-307

[21] Boscolo S and Turitsyn S K 2012 Phys. Rev. A 85043811

[22] Boscolo S, Finot C, Karakuzu H and Petropoulos P 2014 Opt. Lett. 39 438-441

[23] Finot C, Gukov I, Hammani K and Boscolo S 2018 Opt. Fiber Technol. 45 306-312

[24] Pilozzi L, Farrelly F A, Marcucci G and Conti C 2018 Commun. Phys. 157

[25] Frafan C A, Epstein J and Turner D B 2018 Opt. Lett. 43 5166-5169

[26] Närhi M, Salmela L, Toivonen J, Billet C, Dudley J M and Genty G 2018 Nat. Commun. 94923 